\section{A smoothing motion method for high-speed cable-driven parallel camera robots}

\author{
Huiling Wei ${ }^{1, *}$, Yuanying $\mathrm{Qiu}^{2, *}, \mathrm{Yu} \mathrm{Su}^{3}$ and \\ Ying Sheng ${ }^{2}$
}

${ }^{1}$ College of Mechanical and Electrical Engineering, Foshan University, Foshan 528000, China

${ }^{2}$ School of Electro-Mechanical Engineering, Xidian University, Xi'an 710071, China

${ }^{3}$ School of Mechatronic Engineering, Xi' an Technological University, Xi'an 710021, China

This study discusses trajectory planning based on a higher-order polynomial interpolation and the smoothing motion control method of high speed cabledriven parallel camera robots. High speed and high manoeuverability of cable-driven parallel robots make it difficult to achieve stable flexible starting and fast reversing motion. In the existing literature, smoothing motion methods for high-speed cable-driven parallel robots are not adequately considered. For this purpose, first, the transition trajectories of start-stop and turning of the cable-driven parallel robots are planned based on a higher order polynomial interpolation. Further, on the basis of the system dynamic model and the PD feed-forward controller, a disturbance observer is applied in order to inhibit the disturbances from trajectory mutation. Then the stability of the hybrid control strategy is proved. Finally, the main factors which influence the smoothing motion are discussed using numerical simulations and experimental tests. The results indicate that the smoothing motion method consisting of higher-order polynomial interpolation and the disturbance observer are both effective and feasible for engineering applications.

Keywords: Camera robots, hybrid control strategy, stability analysis, transition trajectory.

CABLE-DRIVEN parallel camera robots are a kind of parallel mechanisms whose end-effectors are driven through a specific number of cables. They take some outstanding advantages over the rigid parallel robots such as large workspace, simple structure, small inertia and high payload-to-weight ratio. Due to these characteristics, some cable-driven parallel camera robots have been applied in wind tunnel experiments, medical rehabilitation, astronomical observations, material transportation and other fields ${ }^{1-6}$.

With the growth of TV broadcast technology, the demands of aerial photography are fast increasing. The helicopter aerial camera and rocker camera are adopted as traditional methods of aerial panoramic photographing.

*For correspondence. (e-mail: weihuiling2007@126.com; yyqiu@mail.xidian.edu.cn)
However, the helicopter aerial camera has some disadvantages such as instability and noise. It is also easily influenced by weather. The rocker camera has a small workspace because of its limited freedom and lift height. The rocker camera may also interrupt attention of audience. Hence, the large-scale aerial panorama can be achieved perfectly by cable-driven parallel camera robots $^{7}$. But high speed and high manoeuverability of cable-driven parallel camera robots cause difficulties in stable flexible starting and fast reversing motion. Hence, it is a challenge to find a smooth and stable motion method on the cable-driven parallel camera robots.

Figure 1 illustrates the structure of the cable-driven parallel camera robots. It consists of a camera platform, four drive cables, four fixed pulleys, four servo motors and four masts. The camera platform is connected to the fixed pulleys by cables. The pulleys are attached to the mast ceiling. Four servo motors are fixed on the floor. The cables can be freely deployed and retractable. As shown in Figure 1, the camera platform can be assumed as a point mass. A detailed explanation can be found in Wei et $a l^{8}$. Hence, the camera platform can operate freely with three translational degrees of freedom in the workspace. Because the camera platform is characterized by high speed and high manoeuverability, its motion characteristics are different from the general cable-driven parallel robots, and it is necessary to solve the motion stability problem.

Some researchers have worked on the motion stability control of cable-driven parallel robots. Kawamura ${ }^{9}$ describes a wire-driven parallel manipulation system named FALCON. But it is different from the cable-driven parallel camera robots. FALCON is an over-constrained mechanism which uses more than $n+1$ cables to realize the movement of $n$ degrees of freedom. Oh et al. ${ }^{10}$ propose a feedback control law for cable-suspended robots based on control Lyapunov functions. However, the control method is under input constraints. Carricato and Merlet $^{11}$ propose an optimization algorithm to analyse the

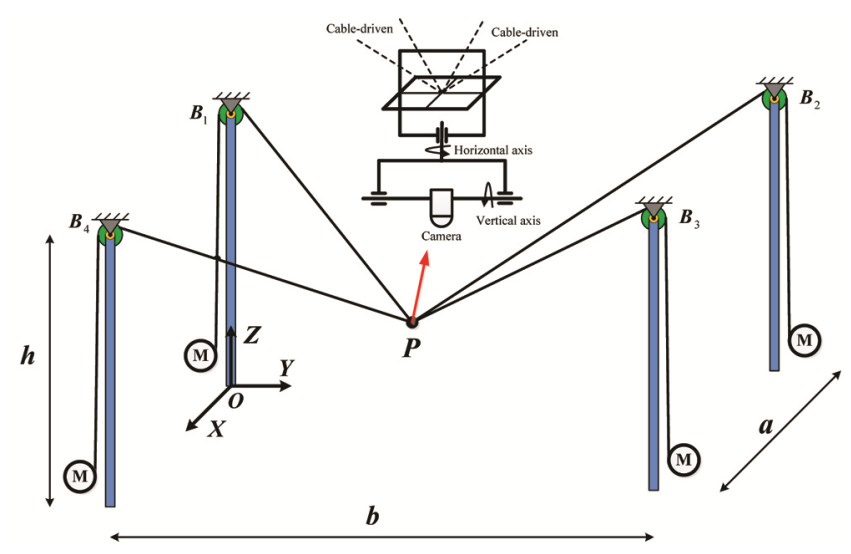

Figure 1. Structural diagram of the cable-driven parallel camera robots. 
stability of the unconstrained cable-driven parallel robots. But, this study is limited to the unconstrained cable-driven parallel robots in static balance. Khosravi and Taghirad ${ }^{12}$ present a robust PID controller for the fully constrained planar cable-driven parallel manipulators. The stability of the closed-loop system is discussed using Lyapunov theory and it shows that through selecting the suitable controller gains, the closed-loop system would be stable. But there is no discussion on high-speed motion. Du and co-workers ${ }^{13,14}$ adopt a variable domain finite element method to establish a dynamic model of cable-driven parallel robots. A controller for restraining the vibration of long cables is designed based on this model. But this cable-driven parallel robot moves slowly. Liu et al. ${ }^{15}$ put forward three performance indices to explain the distribution of minimum cable tension in the workspace. The three performance indices could evaluate the stability of a cable-driven parallel robot. But only the structural stability is studied. Motion stability is not involved. Su and co-workers ${ }^{16,17}$ deal with the continuous and real-time cable tensions of cable-driven parallel robots. But they have not analysed the stability. In our previous research works ${ }^{8,18}$, we have studied the structural stability and motion stability. We used PD forward controller to analyse the stable-tracking performances. However, we did not involve the transition trajectory planning of start-stop and turning, and also, did not use the disturbance observer to inhibit disturbances from trajectory mutation. Here, we adopt a hybrid controller with PD feed-forward control and a disturbance observer based on transition trajectory planning to achieve a smoothing stable motion for cable-driven parallel camera robots with high speed.

As mentioned above, the existing literature does not include a comprehensive method for motion stability of the cable-driven parallel robots. Motion characteristics of cable-driven parallel camera robots are high speed and high manoeuverability. This leads to much higher requirements of maximum acceleration and rising time. Hence, in this study we discuss the motion stability problem of cable-driven parallel camera robots with high speed and high manoeuverability.

The trajectories are the time histories of velocity, acceleration and position. The trajectory planning can be defined such that the cable-driven parallel robots run according to the job requirements along a desired trajectory from the initial state to the end state ${ }^{19}$. The objective of the trajectory planning is to make a reasonable arrangement for the velocity and acceleration, which makes continuous and smooth tracking for the cabledriven parallel camera robots.

It is difficult to operate smoothly when a cable-driven parallel camera robot is at start-stop and turning stages. Because of the large acceleration, this leads to vibrations of the cables. Hence, it is necessary to take measures to make the cable-driven parallel robots work stably and smoothly at the start-stop and turning stages. Making a suitable transition trajectory planning of cable-driven parallel camera robots is an effective method.

The trajectory should be clear of the cable-driven parallel camera robots because of high manoeuverability in the tracking process. More important, velocity, acceleration and cable tension have certain requirements when the cable-driven parallel camera robots are at start-stop and turning stages. So a higher-order polynomial interpolation method is required to satisfy the application requirements. To eliminate the non-smooth trajectory at the start-stop and turning stages, a higher-order polynomial interpolation is carried out for motion transition trajectory planning in this study.

We can use the higher-order polynomial interpolation method to make the variable motion stable. Assume the starting speed of the start-stop or turning stage to be $v\left(t_{0}\right)$, and the end speed of the start-stop or turning stages to be $v\left(t_{\mathrm{f}}\right)$. To guarantee that the velocity, acceleration, and cable tension are continuous and smooth at the $t_{0}-t_{\mathrm{f}}$ period, a fifth-order polynomial interpolation method is necessary to plan the motion transition trajectory. It can be formulated as follows ${ }^{19}$

$$
S(t)=a_{0}+a_{1} t+a_{2} t^{2}+a_{3} t^{3}+a_{4} t^{4}+a_{5} t^{5} .
$$

The coefficients of the polynomials satisfy the following six constraint conditions ${ }^{19}$

$$
\begin{aligned}
& S_{0}=a_{0}+a_{1} t_{0}+a_{2} t_{0}^{2}+a_{3} t_{0}^{3}+a_{4} t_{0}^{4}+a_{5} t_{0}^{5}, \\
& S_{\mathrm{f}}=a_{0}+a_{1} t_{\mathrm{f}}+a_{2} t_{\mathrm{f}}^{2}+a_{3} t_{\mathrm{f}}^{3}+a_{4} t_{\mathrm{f}}^{4}+a_{5} t_{\mathrm{f}}^{5}, \\
& \dot{S}_{0}=a_{1}+2 a_{2} t_{0}+3 a_{3} t_{0}^{2}+4 a_{4} t_{0}^{3}+5 a_{5} t_{0}^{4}, \\
& \dot{S}_{\mathrm{f}}=a_{1}+2 a_{2} t_{\mathrm{f}}+3 a_{3} t_{\mathrm{f}}^{2}+4 a_{4} t_{\mathrm{f}}^{3}+5 a_{5} t_{\mathrm{f}}^{4}, \\
& \ddot{S}_{0}=2 a_{2}+6 a_{3} t_{0}+12 a_{4} t_{0}^{2}+20 a_{5} t_{0}^{3}, \\
& \ddot{S}_{\mathrm{f}}=2 a_{2}+6 a_{3} t_{\mathrm{f}}+12 a_{4} t_{\mathrm{f}}^{2}+20 a_{5} t_{\mathrm{f}}^{3} .
\end{aligned}
$$

where $S$ is an arc coordinate of the trajectory, the parameters $S_{0}, S_{\mathrm{f}}, \dot{S}_{0}, \dot{S}_{\mathrm{f}}$ and $\ddot{S}_{0}, \ddot{S}_{\mathrm{f}}$ are determined by the boundary conditions of the position, velocity and acceleration at the starting or ending points. From eq. (2), we can observe that the transition trajectory planning can effectively inhibit the increasing acceleration indefinitely at the starting or ending points based on the constraint. It mitigates the vibrations of the cables, and then improves the stable motion of the end-effector. When $t_{0}=0$, the solutions to the above linear equations are as follows ${ }^{19}$

$$
a_{0}=S_{0} \text {, }
$$




$$
\begin{aligned}
& a_{1}=\dot{S}_{0}, \\
& a_{2}=\frac{\ddot{S}_{0}}{2}, \\
& a_{3}=\frac{20 S_{\mathrm{f}}-20 S_{0}-\left(8 \dot{S}_{\mathrm{f}}+12 \dot{S}_{0}\right) t_{\mathrm{f}}-\left(3 \ddot{S}_{0}-\ddot{S}_{\mathrm{f}}\right) t_{\mathrm{f}}^{2}}{2 t_{\mathrm{f}}^{3}}, \\
& a_{4}=\frac{30 S_{\mathrm{f}}-30 S_{0}-\left(14 \dot{S}_{\mathrm{f}}+16 \dot{S}_{0}\right) t_{\mathrm{f}}-\left(3 \ddot{S}_{0}-2 \ddot{S}_{\mathrm{f}}\right) t_{\mathrm{f}}^{2}}{2 t_{\mathrm{f}}^{3}}, \\
& a_{5}=\frac{12 S_{\mathrm{f}}-12 S_{0}-\left(6 \dot{S}_{\mathrm{f}}+6 \dot{S}_{0}\right) t_{\mathrm{f}}-\left(\ddot{S}_{0}-\ddot{S}_{\mathrm{f}}\right) t_{\mathrm{f}}^{2}}{2 t_{\mathrm{f}}^{3}} .
\end{aligned}
$$

In order to highlight the advantages of the higher-order polynomial interpolation method, we will make a comparison among parabolic interpolation planning, cubic polynomial interpolation planning and higher-order polynomial interpolation method.

The effects of the cable mass on the dynamic model must be taken into account for large-span cables ${ }^{20-22}$. A detailed derivation process of the cable model with timevarying length is available in the literature ${ }^{16-18}$. The cable model with time varying length is presented as follows

$$
\rho \sum_{j=1}^{N} L_{i} S=T_{i}-\rho g L_{i} \quad(i=1, \ldots, 4)
$$

where $\rho$ is the line density of the cable, $L_{i}$ the cable length, $S$ the position vector of any node on the cable, $T_{i}$ the cable tension and $g$ is the acceleration due to gravity.

The cables and end-effector are regarded as a whole. Hence, the dynamic equation of the research object in the global coordinates is expressed as

$$
\begin{aligned}
& M(X) \ddot{X}+N(X, \dot{X}) \dot{X}+P(X) M_{\mathrm{c}} \\
& \quad+F_{\mathrm{g}}+W_{\mathrm{g}}-W_{\mathrm{e}}=J H,
\end{aligned}
$$

where $M(X)$ are the mass-related items, $\ddot{X}$ the generalized accelerations of the end-effector, $N(X, \dot{X})$ the velocity-related items, $\dot{X}$ the generalized velocities of the end-effector, $P(X)$ the cable inertia related terms, $M_{\mathrm{c}}$ the cable mass, $F_{\mathrm{g}}$ the gravitational forces of the cablerelated terms, $W_{\mathrm{g}}$ the gravitational forces of the endeffector, $W_{\mathrm{e}}$ the generalized external forces exerted on the end-effector centre point by the environment, $H$ the vectors describing horizontal components of all the cable tensions and $J$ is a Jacobian matrix.

The actuator of the cable-driven parallel robots includes a motor, speed gears and winches. The actuator dynamic equation is expressed $\mathrm{as}^{23}$
$\tau=A \ddot{\varphi}+C \dot{\varphi}+r T$,

where $\tau$ are the torques of the motors, $A$ the inertia matrix, $C$ the viscous friction, $r$ the winch radius, $\varphi$ the angles of the motor and $T$ is the cable tension.

Since $T=H \sqrt{1+\tan ^{2} \gamma}$ (ref. 18), the dynamic model of the cable-driven parallel robots is given by

$$
M_{\text {eq }}(X) \ddot{X}+N_{\text {eq }}(X, \dot{X}) \dot{X}+G_{\text {eq }}(X)=J^{T} \tau,
$$

where

$$
\left\{\begin{aligned}
M_{\mathrm{eq}}(X)= & r \sqrt{1+\tan ^{2} \gamma} M(X)+r^{-1} J^{T} A J, \\
N_{\mathrm{eq}}(X, \dot{X})= & r \sqrt{1+\tan ^{2} \gamma} N(X, \dot{X})+r^{-1} J^{T} A \dot{J} \\
& +r^{-1} J^{T} C J \dot{X}, \\
G_{\mathrm{eq}}(X)= & r \sqrt{1+\tan ^{2} \gamma} P(X) M_{\mathrm{c}} \\
& +r \sqrt{1+\tan ^{2} \gamma}\left(F_{\mathrm{g}}+W_{\mathrm{g}}-W_{\mathrm{e}}\right) .
\end{aligned}\right.
$$

Wei et al. ${ }^{18}$ provide a detailed derivation.

Trajectory mutation makes cable-driven parallel camera robots operate unstably. So, using the disturbance observer to inhibit disturbances from trajectory mutation is necessary. Hence, we present a novel combination method to make the cable-driven parallel camera robots operate stably, which is a hybrid controller with PD modified feed-forward controller and a disturbance observer. The role of the PD modified feed-forward controller is to reduce the cable vibration and help the end-effector achieve high-precision tracking. The function of the disturbance observer is to observe the interferences, and then the controller restrains the interferences, and improves the motion stability.

Trajectory mutation reduces the control precision of the cable-driven parallel camera robots in practical applications. In order to solve this problem, we present a hybrid control strategy. The design process is divided into two parts: performance design and interference suppression. As shown in Figure 2, the controller is composed of a PD

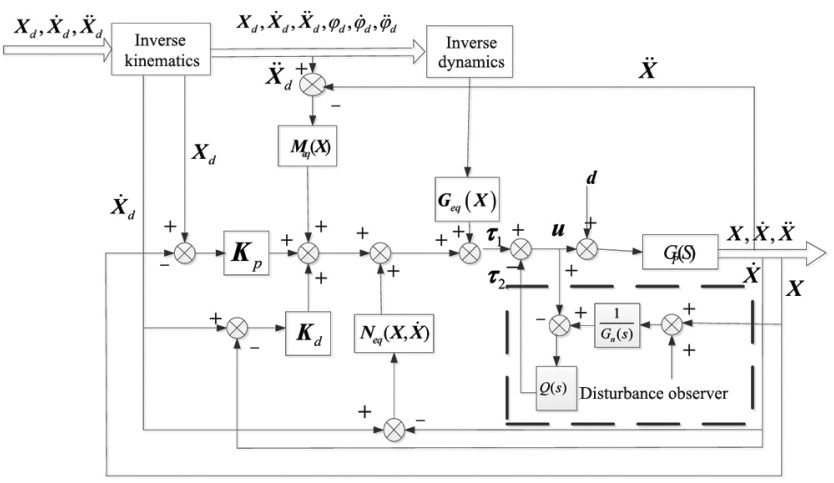

Figure 2. Diagram of the hybrid control system. 
modified feed-forward controller and a disturbance observer. Here, $d$ is the external interference, $G_{\mathrm{n}}(s)$ the nominal model of the DC motor, $G_{\mathrm{p}}(s)$ a practical model of the drive system, and $Q(s)$ is a low pass filter. $K_{\mathrm{p}}$ and $K_{\mathrm{d}}$ are the feedback gain matrices.

According to Figure 2, the control law of the hybrid controller is formulated as follows

$$
u=\tau_{1}+\tau_{2}
$$

Here $\tau_{1}$ is the PD modified feed-forward control law, and $\tau_{2}$ is the compensation control law of the disturbance observer.

According to Wei et al. ${ }^{18}$, the modified PD feedforward control law is given as follows

$$
\begin{aligned}
\tau_{1}= & J^{\dagger}\left(M_{\mathrm{eq}}(X) \ddot{X}_{\mathrm{d}}+N_{\mathrm{eq}}(X, \dot{X}) \dot{X}_{\mathrm{d}}+G_{\mathrm{eq}}(X)\right. \\
& \left.+K_{p}\left(X_{\mathrm{d}}-X\right)+K_{\mathrm{d}}\left(\dot{X}_{\mathrm{d}}-\dot{X}\right)\right)+Q,
\end{aligned}
$$

where $J^{\dagger}$ is the Moore-Penrose generalized inverse ${ }^{18}, Q$ is a zero-dimensional base space, and it guarantees that all cables hold tension. That is to say, it ensures that each cable tension is greater than or equal to the threshold value of the cable tension, avoiding the cable pseudodrag.

The idea of the disturbance observer is that the external interference changes the model parameters and they are equivalent to the input of the controller. In order to achieve the interference mitigation, an equivalent compensation is introduced. Here, we consider trajectory mutation as the generalized interferences. The disturbance observer is used to observe the above interferences. The control of the input side of the compensation is suppressed (Figure 3).

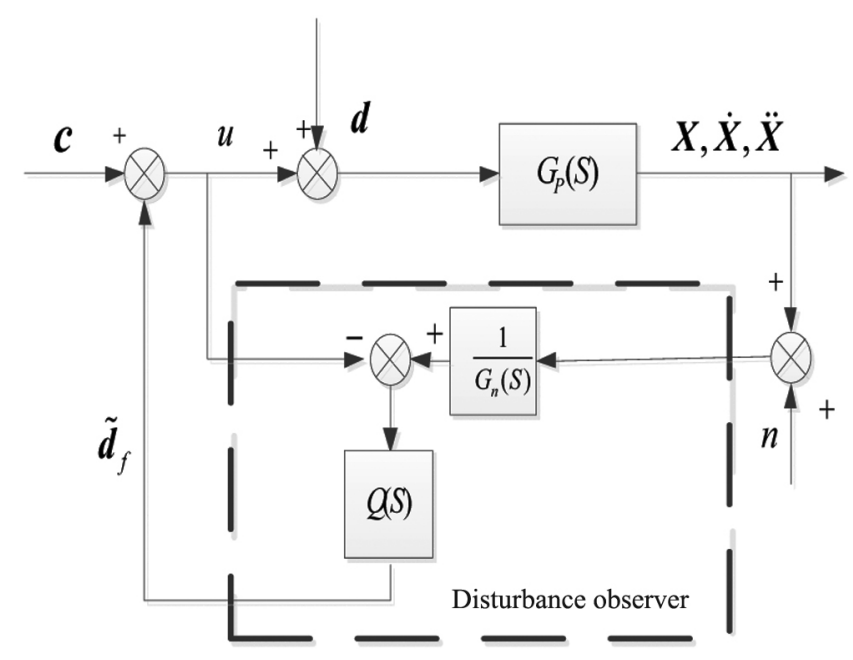

Figure 3. Schematic of the disturbance observer.
Trajectory mutation in the tracking process of a cabledriven parallel camera robot passes through the cables to the drive units, so that there are real-time changes in the motion load. The changing loads are equivalent to the external interferences to the motor, which is expressed by a vector $d, \tilde{d}_{\mathrm{f}}$ is equivalent to the estimated values of $d . n$ is the measurement noise of the motor speed in Figure 3. According to the dynamic model of the driving system, a third-order low pass filter is adopted ${ }^{24}$. The control output of the disturbance observer is described as follows

$$
\tau_{2}=-\tilde{d}_{\mathrm{f}},
$$

It is necessary to ensure the stability of the disturbance observer. So, the low-pass filter is applied to inhibit the interferences and eliminate the noise effect. Based on the robust stability theorem, the sufficient conditions are as follows ${ }^{24}$

$$
\|\Delta(s) Q(s)\|_{\infty} \leq 1
$$

where $\Delta(s)=\left(G_{\mathrm{p}}(s)-G_{\mathrm{n}}(s)\right) / G_{\mathrm{n}}(s)$ (ref. 27). $G_{\mathrm{p}}(s)$ is a practical model of the drive system. Considering the robustness and anti-interference capability, a third-order low pass filter is formulated as follows

$$
Q(s)=\frac{3 \psi s+1}{\psi^{3} s^{3}+3 \psi^{2} s^{2}+3 \psi s+1},
$$

where the value of $\psi$ is determined by $\Delta(s)$ and amplitude frequency characteristics of different bandwidth of $Q(s)$. $s$ is the domain in the Laplasse transforms.

Stability analysis and uniform continuity of eq. (9) are presented in Wei et al. ${ }^{18}$.

The dynamics model has been validated in the literature $^{25,26}$. Therefore, this study focuses on finding the smoothing motion method. The controller, which includes a PD modified feed-forward controller and a disturbance observer, ensures tracking accuracy and stability by the transition trajectory planning of start-stop or turning based on the higher-order polynomial interpolation. Also we focus on analysing the motion stability. In order to prove that the higher-order polynomial interpolation is effective and the control strategy is reasonable, a simulation is studied with distributed mass cables.

The $1 \#$ mast is taken as the coordinate origin. Table 1 shows the simulation parameters of the camera robot.

As shown in Figure 4, take any three points in the workspace as three vertices of the spatial triangle trajectory. The three vertices of the triangle are $p_{1}=$ $\left[\begin{array}{lll}10 & 10 & 5\end{array}\right]^{\mathrm{T}} \mathrm{m}, \quad p_{2}=\left[\begin{array}{lll}30 & 25 & 10\end{array}\right]^{\mathrm{T}} \mathrm{m}, \quad p_{3}=\left[\begin{array}{lll}20 & 20 & 6\end{array}\right]^{\mathrm{T}} \mathrm{m}$ respectively. In the process of simulation, the running time along each edge is $t_{1}=10 \mathrm{~s}, t_{2}=5 \mathrm{~s}, t_{3}=5 \mathrm{~s}$ respectively. A total running time of the spatial triangle trajectory is $20 \mathrm{~s}$. The differential equations are solved for 
Table 1. Simulation parameters of the camera robot

\begin{tabular}{lll}
\hline Parameter & Symbol & \multicolumn{1}{c}{ Value } \\
\hline End-effector mass & $m$ & $25 \mathrm{~kg}$ \\
Position of 1\# pulley & $A_{1}$ & {$\left[\begin{array}{lll}0 & 0 & 24\end{array}\right]^{\mathrm{T}} \mathrm{m}$} \\
Position of 2\# pulley & $A_{2}$ & {$\left[\begin{array}{lll}4 & 0 & 24\end{array}\right]^{\mathrm{T}} \mathrm{m}$} \\
Position of 3\# pulley & $A_{3}$ & {$\left[\begin{array}{lll}40 & 30 & 24\end{array}\right]^{\mathrm{T}} \mathrm{m}$} \\
Position of 4\# pulley & $A_{4}$ & {$\left[\begin{array}{lll}0 & 34 & 24\end{array}\right]^{\mathrm{T}} \mathrm{m}$} \\
Elastic modulus of the cable & $E$ & $2.8 \times 10^{4} \mathrm{MPa}$ \\
Nominal diameter of the cable & $r$ & $3 \mathrm{~mm}$ \\
The linear density of cable mass & $m_{0}$ & $0.456 \mathrm{~kg} / \mathrm{m}$ \\
Maximum threshold value of the cable tension & $T_{\max }$ & $3000 \mathrm{~N}$ \\
Minimum threshold value of the cable tension & $T_{\min }$ & $10 \mathrm{~N}$ \\
Viscous damping coefficient of the cable & $c$ & $0.2 \mathrm{~N} \mathrm{~s} / \mathrm{m}$ \\
Maximum motor rated output torque & $\tau_{\max }$ & $15.8 \mathrm{~N} \mathrm{~m}$ \\
Minimum motor rated output torque & $\tau_{\min }$ & $-15.8 \mathrm{~N} \mathrm{~m}$ \\
Rotational inertia of the motor & $A$ & $7.52 \times 10^{-4} \mathrm{~kg} \mathrm{~m}{ }^{2}$ \\
Viscous damping coefficient of the motor & $C$ & $1.88 \times 10^{-4} \mathrm{~N} \mathrm{~s} / \mathrm{m}$ \\
Reduction ratio of the motor & $n$ & $4: 1$ \\
Radius of the winch & $r_{\mathrm{w}, \mathrm{i}}$ & $0.04 \mathrm{~m}$ \\
\hline
\end{tabular}

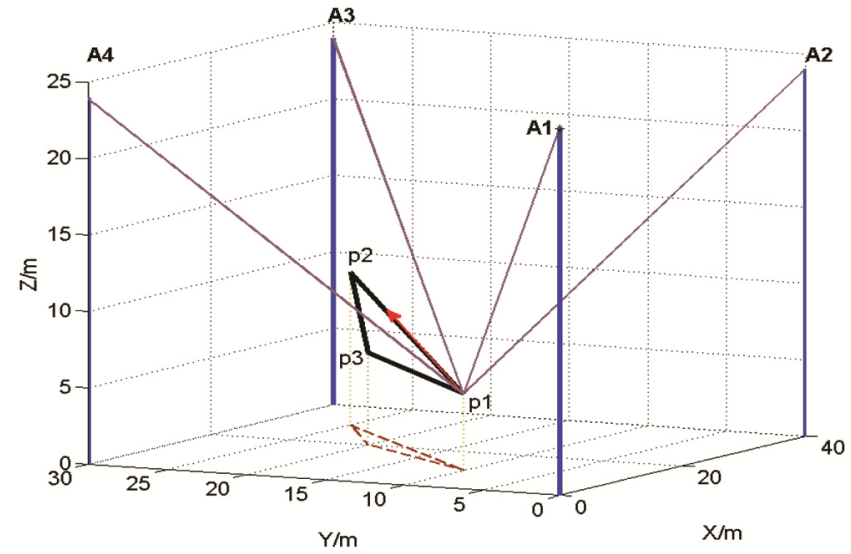

Figure 4. Spatial triangle trajectory.

numerical solution by fourth-order Runge-Kutta. The control parameters $K_{\mathrm{p}}$ and $K_{\mathrm{d}}$ are determined by the critical ratio method ${ }^{27}$.

In order to verify the rationality of start-stop transition trajectory planning and prove the hybrid controller with PD feed-forward controller and a disturbance observer can make the camera robot operate stably, the end-effector runs along the triangle path. The end-effector experiences a start, two turnings and a stop during a loop running. The starting time, first turning time, second turning time and stop time are $t=0 \mathrm{~s}, t=10 \mathrm{~s}, t=15 \mathrm{~s}$ and $t=20 \mathrm{~s}$ respectively. The motion is planned along each line segment of the triangle using the fifth-order polynomial interpolation method from eqs (1) to (3). It is assumed that $\dot{S}_{0}=0, \quad \dot{S}_{\mathrm{f}}=1 \mathrm{~m} / \mathrm{s}, \ddot{S}_{0}=0.01 \mathrm{~m} / \mathrm{s}^{2}, \ddot{S}_{\mathrm{f}}=0 \quad$ during the accelerating stage, and $\dot{S}_{0}=1 \mathrm{~m} / \mathrm{s}, \quad \dot{S}_{\mathrm{f}}=0$, $\ddot{S}_{0}=-0.01 \mathrm{~m} / \mathrm{s}^{2}, \ddot{S}_{\mathrm{f}}=0$ during the deceleration phase.

Figure 5 shows the characteristics of cables through different planning methods for start-stop and turning. As seen in Figure $5 a$ and $b$, cable tensions and cable lengths are not continuous and smooth through parabolic interpolation planning. That shows that the parabolic interpolation planning for start-stop and turning does not meet the motion requirements. It can be seen from Figure $5 a$ that the cable tension mutation appears at the midpoint of movement time and at the turning points. The cable length mutation appears at the midpoint of movement time (Figure $5 b$ ). These phenomena depend on the property of the parabolic interpolation planning. Because the second derivative of parabolic interpolation over time is constant, that is, the magnitude of acceleration is constant, there is continuous transition of velocity and position. However, the end-effector moves from uniform acceleration to uniform deceleration at the midpoint of movement time, and its direction of acceleration changes abruptly. This leads to a sudden change in the cable tension, and so there is a sudden change in cable tension and cable length (Figure $5 a$ and $b$ ).

From Figure $5 c$ and $d$, it is seen that cable tension and cable length are not continuous and smooth through cubic polynomial interpolation planning. What is worse, there are jumps at the start-stop and turning stages. That is, cubic polynomial interpolation planning is not an effective method for transition trajectory planning for cabledriven parallel camera robots. The cubic polynomial interpolation planning is a simple polynomial interpolation method. Only position and velocity of the endeffector are constrained when given the start point and target point. However, the working condition of the cable-driven parallel camera robot is complicated and changeable, and its motion constraints are stricter. The cubic polynomial interpolation planning fails to take the constraints of acceleration and acceleration derivative into account. Therefore, the trajectory planned by it will lead to sudden changes in acceleration, and the trajectory 


\section{RESEARCH COMMUNICATIONS}
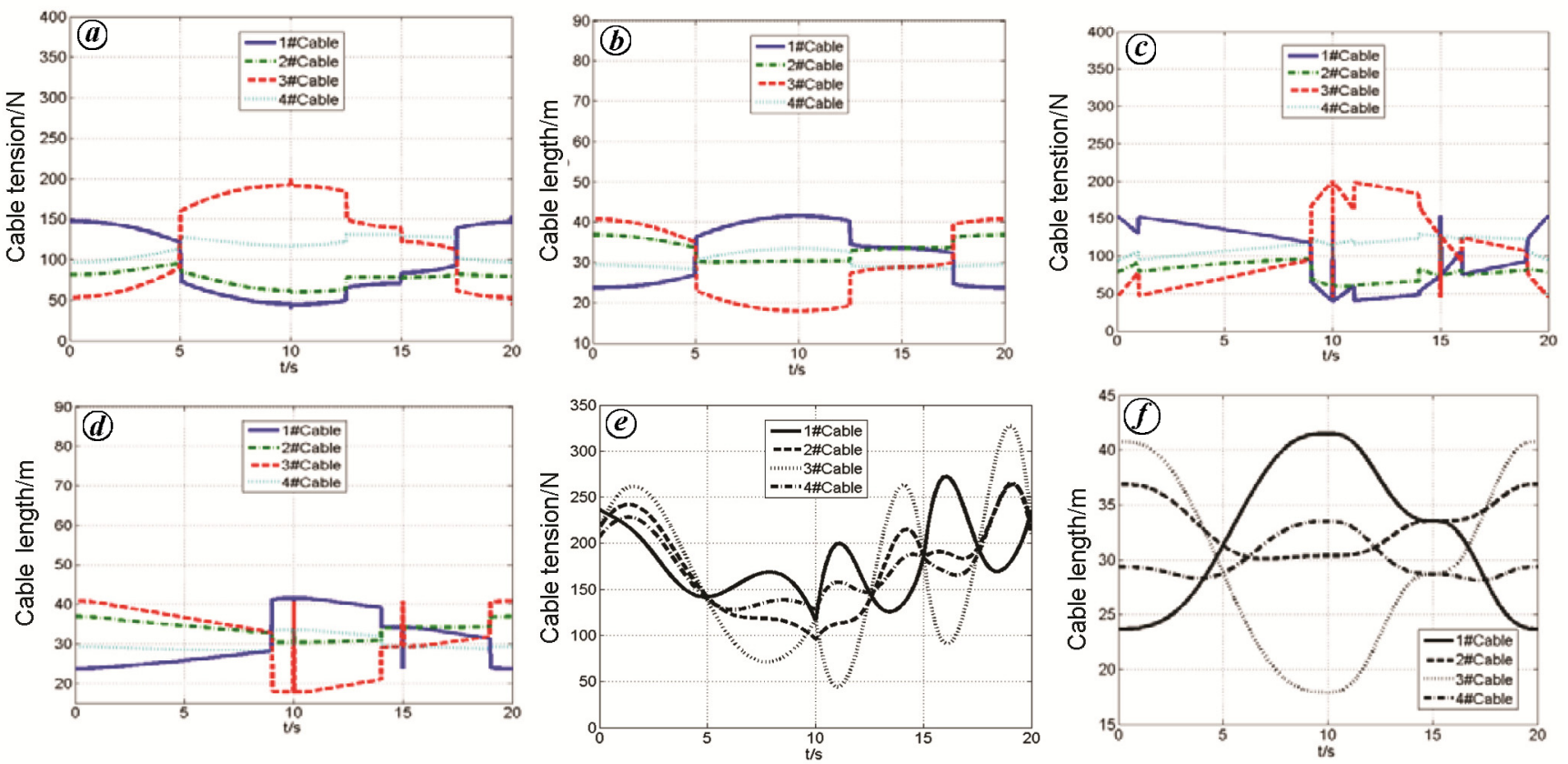

Figure 5. Cable characteristics with different planning methods. $\boldsymbol{a}$, Cable tension through parabolic interpolation. $\boldsymbol{b}$, Cable length through parabolic interpolation. $\boldsymbol{c}$, Cable tension through cubic polynomial interpolation. $\boldsymbol{d}$, Cable length through cubic polynomial interpolation. $\boldsymbol{e}$, Cable tension through fifth-order polynomial interpolation. $f$, Cable length through fifth-order polynomial interpolation.
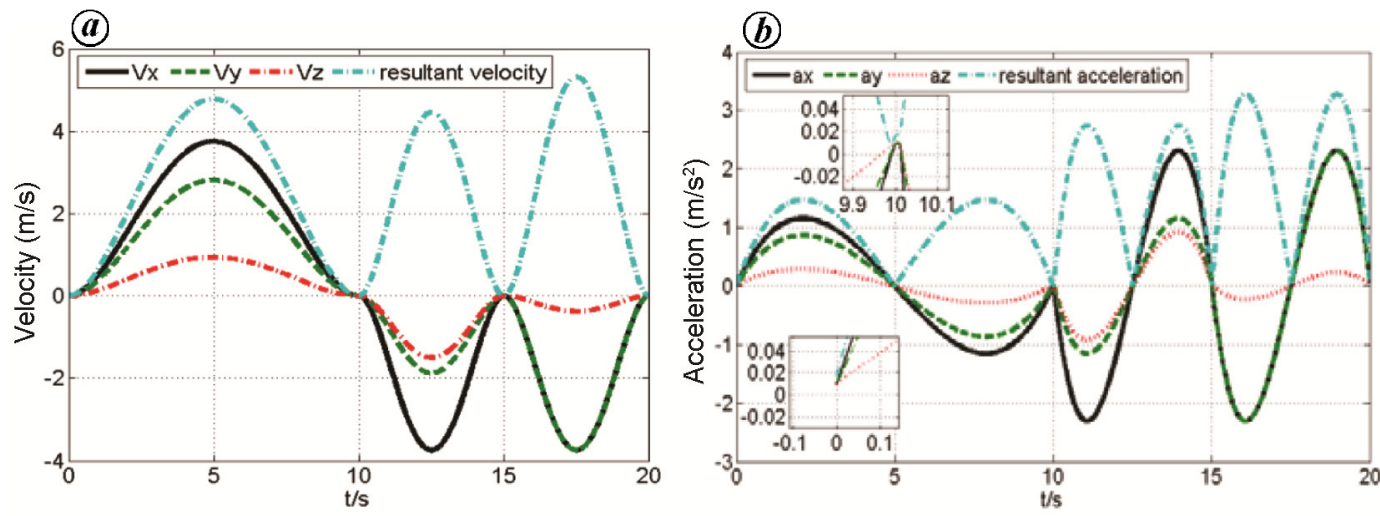

Figure 6. Motion characteristics through fifth-order polynomial interpolation planning. $\boldsymbol{a}$, Velocity; $\boldsymbol{b}$, acceleration.

curve is not smooth. Hence, the cable tension and cable length are also not smooth. The cable-driven robot will not be able to complete the desired exercise. Abrupt changes in acceleration can lead to increasing endeffector jitter and damage the structure of the robot. Therefore, it is necessary to take a higher-order polynomial, which considers and restricts the position, velocity, acceleration and acceleration derivatives simultaneously. Higher-order polynomial interpolation can obtain a more stable trajectory and dynamic characteristics. Thereby it ensures the stability of the cable-driven robot and reduces the occurrence of jitters.

The variations of cable tension in the whole operation process are depicted in Figure $5 e$ through fifth-order polynomial interpolation planning. One may observe that cable tension is continuous in the whole motion process. It is worth noting that there are inflection points at the turning points $(t=10 \mathrm{~s}$ and $t=15 \mathrm{~s})$. The higher-order polynomial interpolation has constraints on position, velocity and acceleration of the end-effector in order to prevent sudden changes in acceleration. The velocity and acceleration of the end-effector are reduced to zero in order to avoid jumping at the inflection points of the triangle. This ensures not only continuous speed, but also continuous acceleration. Then we can get continuous smooth cable tension and cable length. As shown in Figure $5 f$, the variations of cable length are continuous and smooth. There is no jitter phenomenon at the first turning time $t=10 \mathrm{~s}$ and the second turning time $t=15 \mathrm{~s}$. It is a smooth transition, which shows that there is no sway for the end-effector at the two inflection points.

It is also necessary to compare the computational time of the different interpolation methods. It has an important effect on the real-time control. The running time rather 


\section{RESEARCH COMMUNICATIONS}
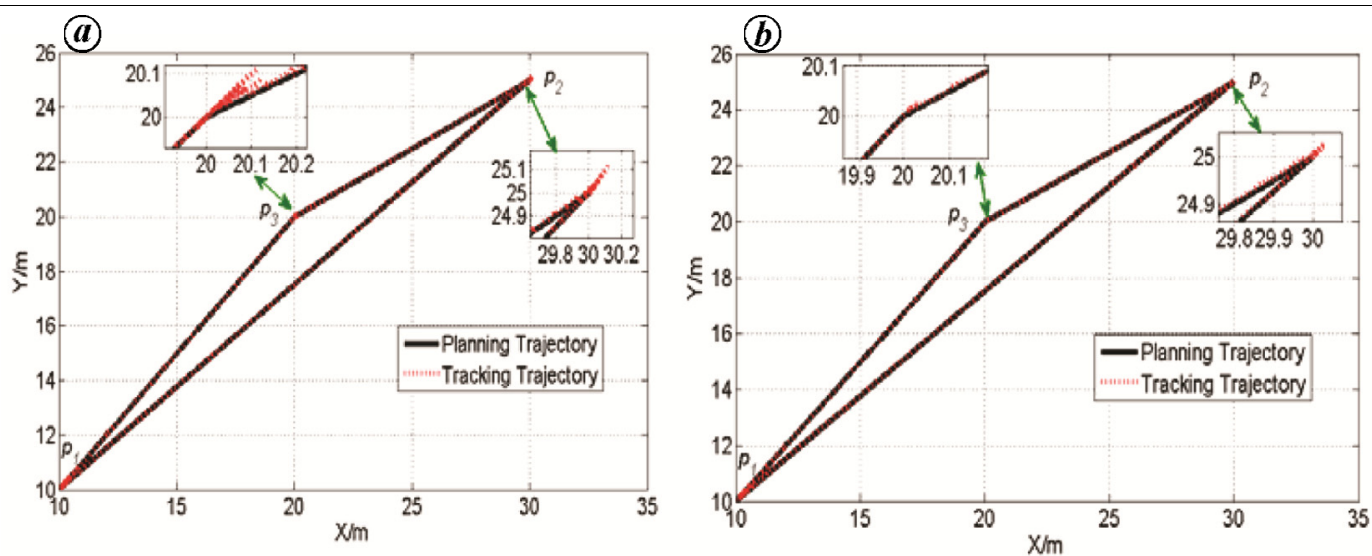

Figure 7. Comparison of trajectory tracking. $\boldsymbol{a}$, Without transition trajectory planning for start-stop and turning; $\boldsymbol{b}$, With transition trajectory planning for start-stop and turning.

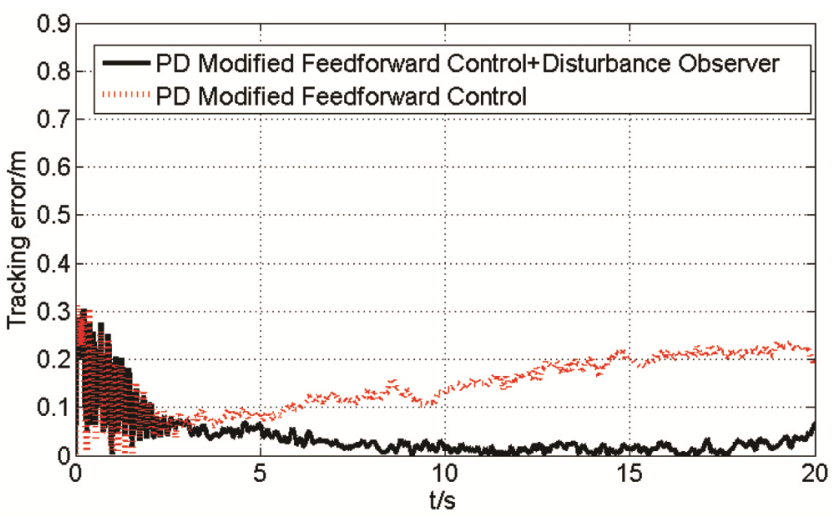

Figure 8. Tracking errors comparison of different control strategies.
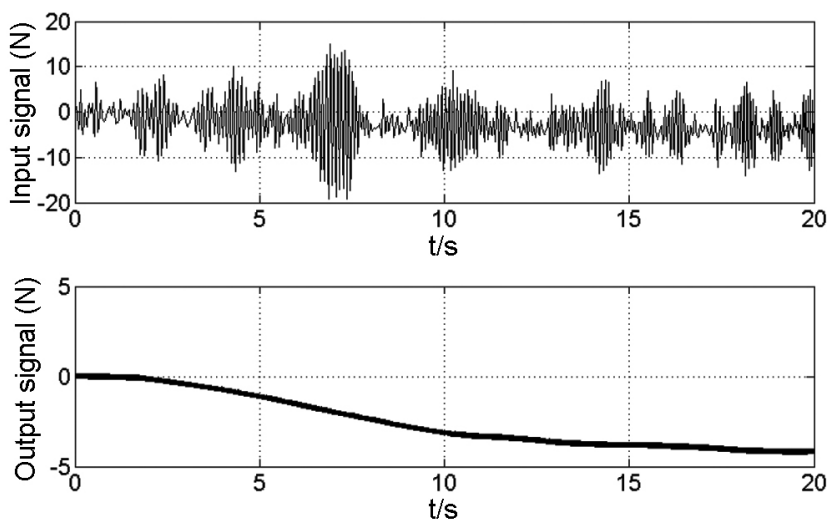

Figure 9. Signal comparison of low-pass filter.

than the computational time along each edge different interpolation methods are as follows: the computational time of parabolic interpolation planning is $0.33 \mathrm{~s}$, the computational time of cubic polynomial interpolation planning is $0.48 \mathrm{~s}$, and the computational time of fifthorder polynomial interpolation planning is $0.68 \mathrm{~s}$. The running time along each edge is $t_{1}=10 \mathrm{~s}, t_{2}=5 \mathrm{~s}, t_{3}=5 \mathrm{~s}$. According to the length of the triangle, the velocity of each edge is $2.55 \mathrm{~m} / \mathrm{s}, 4.54 \mathrm{~m} / \mathrm{s}, 2.84 \mathrm{~m} / \mathrm{s}$ respectively. Comparing the running time with the computational time of fifth-order polynomial interpolation planning, it is worth noting that the running time is ten times more than the computational time. Therefore, the computational efficiency of fifth-order polynomial interpolation planning is able to meet the requirements of real-time control.

Figure 6 depicts the motion characteristics of endeffector through fifth-order polynomial interpolation planning for start-stop and turning. One may observe that the velocity and acceleration of the end-effector are continuous and smooth in the motion process. There is no vibration at the turning points. As seen in Figure $6 a$, the change law of the velocity curves increases first and then decreases; it drops to zero at the turning points. It is evident that the motion achieves a smooth transition of velocity at the two inflection points. The velocity curve indicates that the fifth-order polynomial interpolation planning is reasonable and effective. It is worth noting in Figure $6 b$ that the acceleration increases close to zero at the starting point $t=0 \mathrm{~s}$ and the first turning $t=10 \mathrm{~s}$. It can be seen that the acceleration does not mutate at the turning points. The resultant acceleration is zero when the end-effector passes through the mid-points of the three sides. That is the acceleration values are zero when the time are $5 \mathrm{sec}, 12.5 \mathrm{sec}$ and $17.5 \mathrm{sec}$ respectively. This is because the end-effector accelerates from the beginning and decelerates when it is close to the turning point. In other words, velocity and acceleration are both smooth and continuous after fifth-order polynomial interpolation planning.

In order to analyse the tracking performances after transition trajectory planning, there is a comparison of trajectory planning for start-stop and turning. Figure 7 depicts the comparison of tracking trajectories. The horizontal projection of tracking trajectory without higher-order polynomial interpolation planning for the spatial triangle trajectory is shown in Figure $7 a$, and that with the higher-order polynomial interpolation planning is depicted in Figure $7 b$. As seen in Figure $7 a$, the tracking trajectory has a mutation at the turning points, and does not satisfy continuous and smooth tracking. The 


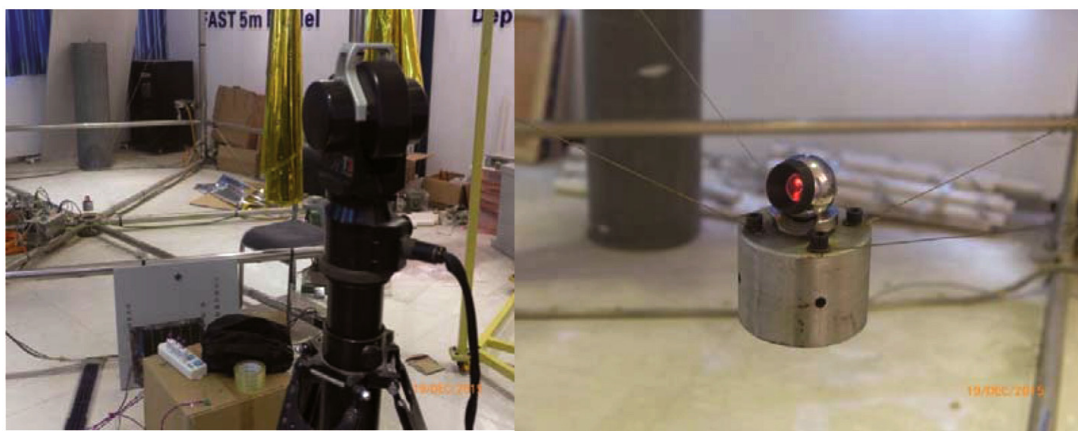

Figure 10. Five metre model for an experimental test.
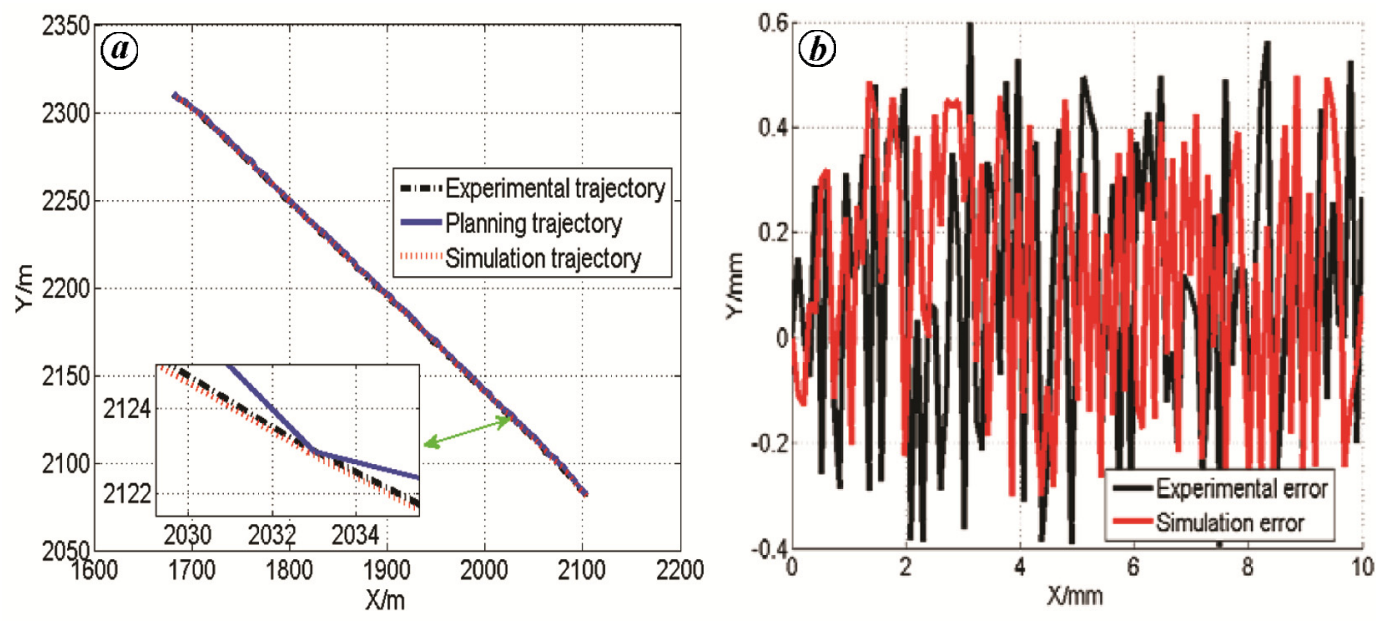

Figure 11. Trajectory tracking (a) and error comparison (b) for $5 \mathrm{~m}$ model.

tracking error is more obvious than that of higher-order polynomial interpolation planning as shown in Figure $7 a$. Tracking trajectory errors without higher-order polynomial interpolation planning at the turning points are 0.1138 and $0.1111 \mathrm{~m}$ respectively. While tracking errors with higher-order polynomial interpolation planning at the turning points are 0.0129 and $0.0159 \mathrm{~m}$ respectively. It can be seen from Figure $7 b$ that there is no jumping at the first and second turning points respectively. The endeffector transits smoothly with smaller tracking error. It is well known that the end-effector achieves continuous smooth trajectory through interpolation planning. This is conducive to the high-speed motion and reduces the vibration of the end-effector. From the above it can be concluded that the higher-order polynomial interpolation planning is effective and reasonable.

In order to analyse the tracking performance of the hybrid controller with the PD feed-forward control and a disturbance observer after transition trajectory planning, we make a comparison between using PD feed-forward control strategy with disturbance observer and PD feedforward control strategy without disturbance observer. Figure 8 shows their position tracking errors. As seen in, the tracking errors of PD feed-forward controller with disturbance observer are smaller than those of the PD feed-forward controller without disturbance observer.
The mean difference of tracking errors of the PD feedforward controller with the disturbance observer is about $0.04 \mathrm{~m}$, while the mean difference of tracking errors of the PD feed-forward controller without the disturbance observer is about $0.16 \mathrm{~m}$. It shows that the disturbance observer can restrain the disturbance from trajectory mutation and improve the stability of motion.

Figure 9 shows a comparison between the input signal and output signal of the low-pass filter. Zero is the set threshold, and only the signals which are below the set threshold can pass normally. It can be seen that the filter can effectively remove noise from the measurements. It is worth noting that the output signal $-\tilde{d}_{f}$ tends to a stationary value after $10 \mathrm{sec}$, which means that the disturbance observer is robust and stable. Hence, design of the low-pass filter $Q(s)$ is reasonable and effective.

In order to verify the validity of transition trajectory planning of start-stop and turning stages, an experimental test was carried out using a $5 \mathrm{~m}$ model. This five meter model uses four cables to manoeuver the end-effect, which includes four servo drives, four servo motors, four cables and the end-effector. The speed control mode is adopted in this model. However, because of the limited workspace of the model, it is only suitable to validate the principle of the control mode, which cannot be used for high-speed experiments. We used the API laser tracker 
to measure the displacement of the end-effect (Figure 10).

Let the camera robot run along the planned space triangle, and we use the API instrument to collect data along the actual running trajectory of the camera robot. Figure $11 b$ is the trajectory tracking error of Figure $11 a$. As shown in Figure $11 b$, the error is not particularly large at the start-up stage. It is also clear from the figure that the maximum error of the experimental trajectory is $0.6 \mathrm{~mm}$, the mean error of the experimental trajectory is $0.31 \mathrm{~mm}$, the maximum error of the simulated trajectory is $0.5 \mathrm{~mm}$, and the mean error of the simulated trajectory is $0.24 \mathrm{~mm}$.

Long cables are prone to vibration at start-stop and turning transition stages when the cable-driven parallel camera robots move with high speed and high manoeuverability. In order to achieve continuous and smooth tracking, a higher-order polynomial interpolation on the motion transition trajectory planning is presented for cable-driven parallel camera robots. It can be noted from the numerical example that the tracking errors with higherorder polynomial interpolation planning reduced one order of magnitude compared with those that without higher-order polynomial interpolation planning. The endeffector achieves continuous and smooth trajectory through the interpolation planning.

Also, in order to inhibit the disturbances from trajectory mutation, a hybrid controller with the PD modified feed-forward controller and a disturbance observer were established to ensure high-speed stable motion. From the numerical example, it is noteworthy that the tracking errors of PD feed-forward controller with the disturbance observer are smaller than that of the PD feed-forward controller without the disturbance observer. The endeffector achieves stable motion with high speed. That is to say, the control strategy can improve the stability of the operation.

There is a $5 \mathrm{~m}$ physical model for a cable-based parallel camera robot in our laboratory at present. Our speed control mode has been adopted in this model. However, because of the limited workspace of the model, it is only suitable for low-speed principle verification experiments.

Conflict of interest: The authors declare that there is no conflict of interests.

1. Alikhani, A., Behzadipour, S. and Alasty, L. A., Design of a largescale cable-driven robot with translational motion. Robot. Comput.-Integr. Manuf., 2011, 27(2), 357-366.

2. Mao, Y. and Agrawal, S. K., Design of a cable-driven arm exoskeleton (CAREX) for neural rehabilitation. IEEE Trans. Robot., 2012, 28(4), 922-931.

3. Li, C. D., Yi, J. Q. and Yu, Y., Inverse control of cable-driven parallel mechanism using type-2 fuzzy neural network. Acta Automat. Sin., 2010, 36(3), 459-464.

4. Xiao, Y. W., Lin, Q. and Zheng, Y. Q., Model aerodynamic tests with a wire-driven parallel suspension system in low-speed wind tunnel. Chin. J. Aeronaut., 2010, 23, 393-400.
5. Zi, B. et al., Dynamic modeling and active control of a cablesuspended parallel robot. Mechatronics, 2008, 18, 1-12.

6. Qiu, Y. Y. et al., Elimination of force singularity of the cable and cabin structure for the next generation large radio telescope. Mechatronics, 2002, 12(7), 905-918.

7. Vincent, T. L., Stabilization for film and broadcast cameras. IEEE Control Syst. Mag., 2008, 2, 20-25.

8. Wei, H. L., Qiu, Y. Y. and Yang, J., An approach to evaluate stability for cable-based parallel camera robots with hybrid tension-stiffness properties. Int. J. Adv. Robotic Syst., 2015, 12(185), 1-12.

9. Kawamura, S., Development of an ultrahigh speed robot FALCON using wire drive system. J. Robot. Soc. Jpn., 1997, 15(1), 82-89.

10. Oh, S. R. and Agrawal, S. K., A control Lyapunov approach for feedback control of cable-suspended robots. Proceedings of the IEEE International Conference on Robotics and Automation, Roma, 2007, pp. 4544-4549.

11. Carricato, M. and Merlet, J. P., Stability analysis of under constrained cable-driven parallel robots. IEEE Trans. Robot., 2013, 1(29), 288-296.

12. Khosravi, M. A. and Taghirad, H. D., Robust PID control of fullyconstrained cable driven parallel robots. Mechatronics, 2014, 24, $87-97$.

13. Du, J. L. et al., Dynamic analysis of cable-driven parallel manipulators using a variable length finite element. J. Comput. Nonlinear Dyn., 2015,10, 1-6.

14. Du, J. L. and Agrawal, S. K., Dynamic modeling of cable-driven parallel manipulators with distributed mass flexible cables. J. Vibr. Acoust., 2015, 137, 1-8.

15. Liu, P. et al., On the minimum cable tensions for the cable-driven parallel robots. J. Appl. Math., 2014, article id. 350492, 1-8.

16. Su, Y., Qiu, Y. Y. and Liu, P., Optimal cable tension distribution of the high-speed redundant driven camera robots considering cable sag and inertia effects. Adv. Mech. Eng., 2015, article id. $729020,1-11$

17. Su, Y., Qiu, Y. Y. and Liu, P., The continuity and real-time performance of the cable tension determining for a suspend cabledriven parallel camera robot. Adv. Robot., 2015, 29(12), 743-752.

18. Wei, H. L., Qiu, Y. Y. and Su, Y., Motion control strategy and stability analysis for high speed cable-driven camera robots with cable inertia effects. Int. J. Adv. Robot. Syst., 2016, 13, 1-14.

19. Cai, Z. X., Robotics, Tsinghua University Publishing House, Beijing, China, 2009.

20. Riehl, N. et al., Effects of non-negligible cable mass on the static behavior of large workspace cable-driven parallel mechanisms. In Proceedings of IEEE International Conference on Robotics and Automation, Kobe, Japan, 2009, pp. 2193-2198.

21. Shen, S. Z., Xu, C. B. and Zhao, C., Cable Structure Design, China Architecture \& Building Press, Beijing, China, 2006.

22. Irivne, H. M., Cable Structures, MIT Press, Cambridge, 1981.

23. Liu, J. K., Advanced PID Control and MATLAB Simulation, Electronics Industry Press, Beijing, 2003.

24. Arimoto, S., Control Theory of Non-linear Mechanical Systems, Clarendon Press, Oxford, UK, 1996.

25. Wang, Y. Z., Dynamic Analysis of Cable-driven Parallel Robots with Cable Elastic Vibration, Xidian University, Xi'an, 2012.

26. Qiu, Y. Y., Duan, B. Y. and Wei, Q., Optimal distribution of the cable tensions and structural vibration control of the cable-cabin flexible structure. Struct. Eng. Mech., 2002, 14(1), 39-56.

27. Slotine, J. J. E. and Li, W. P., Applied Nonlinear Control, Prentice Hall, NJ, USA, 1991.

ACKNOWLEDGEMENT. This study is fully supported by the National Key R\&D Program of China (2018YFB1308100) and National Natural Science Foundation of China (No. 51175397).

Received 22 May 2019; revised accepted 17 August 2019

doi: $10.18520 / \mathrm{cs} / \mathrm{v} 117 / \mathrm{i} 12 / 2040-2048$ 